\title{
Triangular rogue wave cascades
}

\author{
David J. Kedziora, Adrian Ankiewicz, and Nail Akhmediev \\ Optical Sciences Group, Research School of Physics and Engineering, The Australian National University, Canberra ACT 0200, Australia
}

(Received 20 June 2012; published 8 November 2012)

\begin{abstract}
By numerically applying the recursive Darboux transformation technique, we study high-order rational solutions of the nonlinear Schrödinger equation that appear spatiotemporally as triangular arrays of Peregrine solitons. These can be considered as rogue wave cascades and complement previously discovered circular cluster forms. In this analysis, we reveal a general parametric restriction for their existence and investigate the interplay between cascade and cluster forms. As a result, we demonstrate how to generate many more hybrid rogue wave solutions, including semicircular clusters that resemble claws.
\end{abstract}

DOI: 10.1103/PhysRevE.86.056602

PACS number(s): 05.45.Yv, 47.20.Ky, 42.65.-k

\section{INTRODUCTION}

An unfortunate fact of life is that extreme events have a tendency to arrive in bunches. This concept is reflected in the well-known proverb "bad things come in threes" and its equivalent, "misfortunes never come singly." Indeed, where one occurrence may be labeled a minor disaster, successive incidents can compound a situation into a complete catastrophe. Furthermore, each event is normally unexpected and always appears "from nowhere." So, given the notorious unpredictability of extreme events, due consideration must be given to any scientific approach that explains how these incidents arise.

Archetypically, the serial nature of rogue waves is exemplified by the oceanic "three sisters" phenomenon, where three large waves appear as successive events. This is no longer merely legend but is now actively discussed in the literature [1-4]. Furthermore, many natural disasters potentially modelled as rogue waves show a repetitive or correlated nature. Hence, the issue naturally arises whether groups of extreme events can be described mathematically and whether they are arranged in a specific pattern. Of course, these are generally complicated questions to answer. Nevertheless, if we start with a certain simple model, such as one involving deep ocean wave evolution, we can write a partial differential equation that describes rogue wave phenomena in the lowest approximation. With this, we can give fundamental solutions that portray a group of distinct rogue waves as sequences of extreme events.

In particular, this approach can be applied to the nonlinear Schrödinger equation (NLSE) [5-7]. This is a well-justified model for deep ocean waves [8-10] as well as many other phenomena in physics [11-14]. The basic Peregrine soliton, a spatiotemporally localized solution of the NLSE, has been considered as a prototype of a first-order rogue wave. It has been studied both theoretically $[15,16]$ and experimentally $[17,18]$. Furthermore, despite being a rough approximation of reality, the NLSE accurately describes physical rogue waves of relatively high order [19].

From this model, it is apparent that second-order rogue waves may appear as a single event [20] or as triplets [21] in space and time. Remarkably, there are no solutions that describe rogue waves appearing as doublets. This fact alone

*djk105@ rsphysse.anu.edu.au tells us that only certain patterns of rogue waves represent legitimate solutions to the NLSE [22]. This interesting observation poses the general question of what patterns are possible when we deal with extreme events of even higher order. Clearly, if we try to explain rogue waves based on linear theory [23-25], the concept of interference should allow any imaginable configuration, including a double peak. However, taking into account the rarity of a single high peak wave, two of them occurring in conjunction would be exceptionally improbable within the constraints of linear theory.

Thus, we utilize nonlinear analysis [8], which has been fruitful in describing not only single events but even higherorder solutions $[6,22,26,27]$. This approach recently led to the discovery of sophisticated circular "atomlike" structures [28], as well as higher-order triangular patterns [29] as an alternative arrangement. Each of these cases is nontrivial and requires special techniques for investigation, such as Wronskian methodology [26,27,30], Schur polynomials [29], or Darboux transformations [31-33]. Even so, the rapid increase of complexity with respect to solution order means that the analytic forms of these rogue wave arrangements become unwieldy beyond the case of a simple rogue wave triplet [21]. It is becoming clear that none of the techniques mentioned above can provide a complete understanding of all the structures that may exist in higher orders.

Nonetheless, in the present work, we have made a further step forward and found a systematic way to generate rogue wave patterns in the form of triangular arrays. Although glimpsed as a special case by Ohta and Yang [29], these structures not only complement previously discovered circular cluster forms [28] but can even be "hybridized" with them to produce new solutions of the NLSE, acting almost like primary elements of a structural basis set. To show this, we use the Darboux transformation method [31-33], which was specifically designed for constructing higher-order solutions of a certain class. The main difficulty here is to find the parameters that control the pattern. Unlike eigenvalues from the inverse scattering transform, these parameters do not automatically arise from standard techniques. They are buried deep within the methodology. Although implicitly related to translations along the spatiotemporal axes, they are not simple and describe the structure as a whole rather than the positioning of individual peaks. Thus, they have to be carefully detailed in the theory to be associated with a certain pattern. 
Here, we show how triangularly arrayed cascade solutions appear in our scheme and prescribe the way they can be generated numerically for any order. The first time these complicated structures become pertinent is at third order, which already offers several patterns, in contrast to the simple triplet at second order. This is because the latter solution can be considered as either a triangular or circular array, while the distinction between the two forms must be made clear in the third-order case. By additionally hybridizing the circular cluster and triangular cascade forms, we can find new legitimate solutions of the NLSE, such as a "claw" structure. Thus, our work opens up another range of possible structures to encounter in nature or to produce in experimental works.

\section{THE THEORY}

We, first, establish the key concepts from the Darboux process [31], modified here to operate on the NLSE. In dimensionless form, the target equation is written as

$$
i \frac{\partial \psi}{\partial x}+\frac{1}{2} \frac{\partial^{2} \psi}{\partial t^{2}}+|\psi|^{2} \psi=0
$$

where the wave function $|\psi(x, t)|$ in Eq. (1) commonly describes the wave envelope. Variables $x$ and $t$ have different physical meanings, depending on traditions in each field. For example, in fiber optic applications [17], the variable $x$ is the distance along the fiber while $t$ is the retarded time in the frame moving with the pulse group velocity. On the other hand, in water wave applications [18,34], $x$ is the dimensionless time while $t$ is the distance in the frame moving with the group velocity. Nevertheless, this difference is largely unimportant, as simple linear transformation involving the group velocity allows us to change the equation and variables from one form to another. However, the choice of scaling factors in front of each term in Eq. (1) is unusually prescient; it is because of this particular choice that rogue wave clusters are spatiotemporally circular [28] and, likewise, the cascades we present below are essentially equilateral.

Applied to the NLSE, the Darboux method allows complicated solutions to be generated as nonlinear superpositions of lower-order forms. This procedure is well established in the literature and detailed specifics can be found elsewhere $[28,33]$. As a conceptual summary, the process creates $n$ independent first-order components, the basic structure of which is uniformly determined by a zeroth-order seeding solution, and these are recursively combined into an order- $n$ wave function. For example, a zero-background seeding input, $\psi_{0}=0$, generates simple solitons as individual first-order components, which then can be combined into multisolitonic structures [32].

More relevant to this work is the case when a plane wave, $\psi_{0}=e^{i x}$, is used as the seeding solution. The resulting firstorder "building blocks" produced by the Darboux method are breather solutions, each with an identifying number $j$ and three unique parameters. Two are simply regarded as shifts from a common origin along the $x$ and $t$ axes, which we label as $x_{j}$ and $t_{j}$, respectively. The third is the eigenvalue $l_{j}$, which controls the shape of the first-order component. As the real part of $l_{j}$ only affects the angle a solution makes with

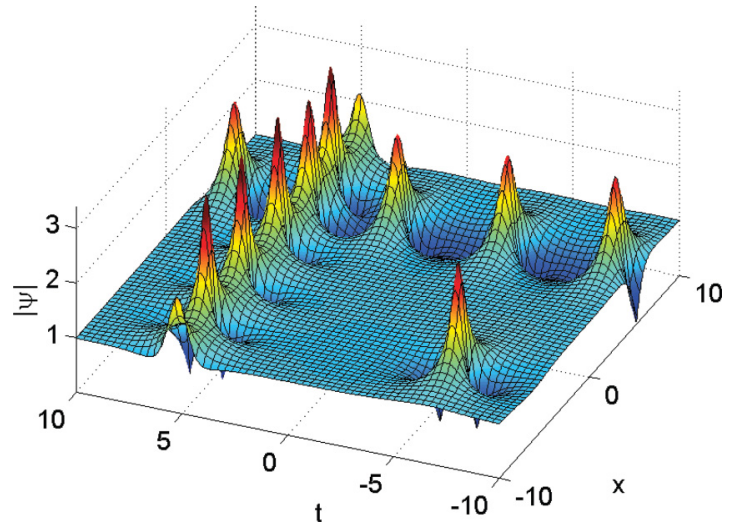

FIG. 1. (Color online) A third-order NLSE solution. Component 1 is an $\mathrm{AB}$ (aligned with the $t$ axis) with parameters $l_{1}=0.8 i, x_{1}=5$, and $t_{1}=0$. Component 2 is a Peregrine soliton with $l_{2} \approx i$ located at $x_{2}=-5$ and $t_{2}=-5$. Component 3 is a $\mathrm{KM}$ soliton (aligned with the $x$ axis) with parameters $l_{3}=1.2 i, x_{3}=0$, and $t_{3}=5$.

respect to the $x$ and $t$ axes, it can be omitted without loss of generality. However, the imaginary part is important for tuning the modulation period of the breather solution, as well as deciding to which axis the wave train is parallel.

For purely imaginary $l_{j}$, we can define the modulation frequency of a component as $\kappa_{j}=2 \sqrt{1+l_{j}^{2}}$. In the case when $0<\operatorname{Im}\left(l_{j}\right)<1\left(\kappa_{j}\right.$ is real), the solution is an Akhmediev breather (AB), which is localized in $x$ but periodic in $t$. When $\operatorname{Im}\left(l_{j}\right)>1$ ( $\kappa_{j}$ is imaginary), the component is a Kuznetsov$\mathrm{Ma}(\mathrm{KM})$ soliton, which is localized in $t$ but periodic in $x$. In the limit of $l_{j} \rightarrow i\left(\kappa_{j} \rightarrow 0\right)$, the period of each solution goes to infinity and, in the first-order scenario, a solitary peak remains. This quasirational solution is named the Peregrine soliton and has gained traction as a prototypical rogue wave. The three structures have been individually displayed previously [28]. In contrast, Fig. 1 here shows the result of the Darboux process for a third-order superposition involving a Peregrine soliton, an $\mathrm{AB}$ and a $\mathrm{KM}$ soliton, all in a single solution. For long periods, both the $\mathrm{AB}$ and $\mathrm{KM}$ soliton can be treated as chains of Peregrine soliton peaks. Indeed, in this limit $\kappa_{j} \rightarrow 0$ for all $j$ ), every well-separated peak appears identical to a Peregrine soliton [17]. Thus, we shall henceforth refer to these as "rogue wave quanta" for convenience.

For the most part, higher-order multibreather solutions of the NLSE are intuitive and easy to comprehend. Although the recursive Darboux method is highly involved, the nonlinear superpositions of individual components are relatively recognizable, as in Fig. 1. However, there are a couple of exceptions to this rule. One is in the limit of two component eigenvalues becoming equal, called the degenerate case, and has been previously investigated in detail [35]. The other is the aforementioned higher-order rogue wave limit, when all component eigenvalues approach $i$. If $x_{j}$ and $t_{j}$ have been left alone, all components are located at the same origin, and the overall wave function appears as a complicated but solitary peak, exemplified by Fig. 2(a). The analytic expression for this solution of eighth order can be written in explicit form [36]. To give an idea of its complexity, we only mention here that it occupies 60 printed pages. When the shift parameters enter 


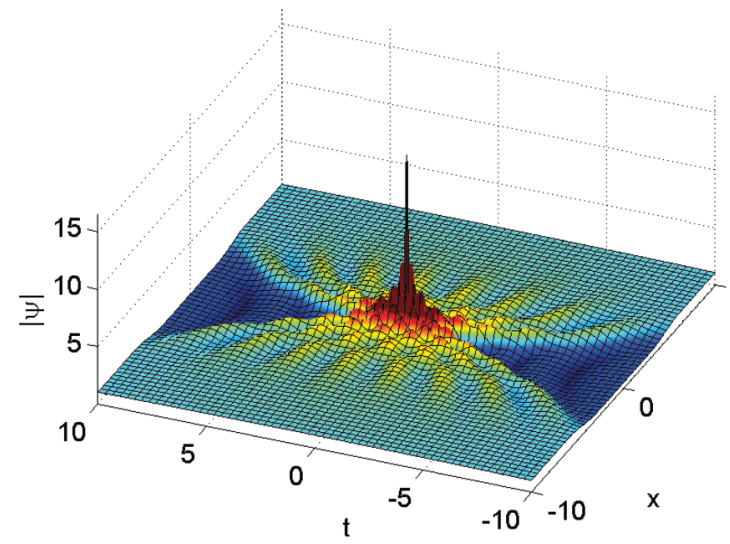

(a)

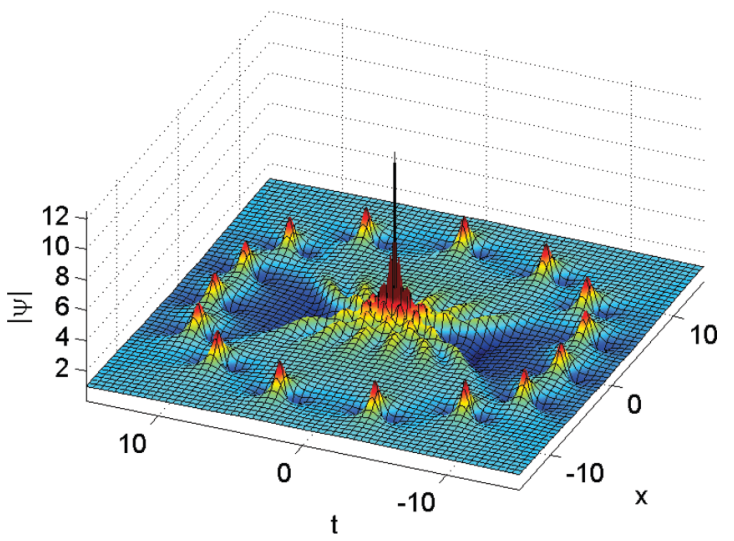

(b)

FIG. 2. (Color online) Eighth-order rogue waves. (a) Unshifted, with all components located at origin. The maximum amplitude is 17, as expected [22]. (b) Shifted, with $X_{18}=5^{14}$.

into play, as in Fig. 2(b), the solution becomes significantly more lengthy.

To avoid unnecessary complications, we henceforth assume component modulation frequencies are ordered by index and are multiples of a common parameter; specifically that $\kappa_{j}=$ $k_{j} \kappa$ and $k_{1}<k_{2}<\cdots<k_{n}$. If we further assume, without loss of generality, that all components are $\mathrm{ABs}$ before the $\kappa \rightarrow 0$ rogue wave limit, then it is known that component $j$ donates $j$ quanta to the resulting structure, such that an order $n$ rogue wave can be considered to consist of $n(n+1) / 2$ quanta [28]. For example, the eighth-order rogue wave in Fig. 2(a) is constructed from eight components but, in reality, it is a fusion of 36 quanta. This is a particularly deep property of NLSE breather solutions, as it indicates that two identical components cannot exist in the same domain without enforcing some asymmetry, essentially by way of contributing unequal numbers of rogue wave quanta to the total solution [35]. The simplest consequence of this property is that no rogue doublet has yet been identified.

Optimally, the way to show the existence of these quanta is to spatiotemporally separate components with the use of the shift parameters $x_{j}$ and $t_{j}$, but this is a nontrivial task. In the $\kappa \rightarrow 0$ limit, any nonzero difference in shift between components is sufficient to repel them from each other to the infinity horizon of the $(x, t)$ plane. However, if component shifts are made dependent on eigenvalue before the $\kappa \rightarrow 0$ limit is applied, according to the relation

$$
\begin{aligned}
& x_{j}=\sum_{m=1}^{\infty} \kappa^{2(m-1)} X_{j m}=X_{j 1}+X_{j 2} \kappa^{2}+X_{j 3} \kappa^{4}+\cdots, \\
& t_{j}=\sum_{m=1}^{\infty} \kappa^{2(m-1)} T_{j m}=T_{j 1}+T_{j 2} \kappa^{2}+T_{j 3} \kappa^{4}+\cdots,
\end{aligned}
$$

where $X_{j m}$ and $T_{j m}$ are "shift expansion" coefficients that are independent of $\kappa$, then it is possible to generate new structures by carefully selecting which coefficients to make nonzero. This is a fundamentally difficult concept to understand due to the intricacies of limit theory; all total shifts are technically zero for $\kappa \rightarrow 0$, provided that $X_{j 1}=T_{j 1}=0$, but the coefficients still survive by a unique form of dissemination into the solution. The result is that, by changing $X_{12}$ (or $T_{12}$ ) alone, a second-order rogue wave becomes a rogue triplet with three quanta pulled apart [21]. Furthermore, for an order $n$ rogue wave, $X_{1 n}$ pulls out a ring of $2 n-1$ quanta, leaving behind a central rogue wave of order $n-2$. This is demonstrated by an unshifted eighth-order rogue wave in Fig. 2(a) becoming a circular rogue wave cluster in Fig. 2(b), with 15 peaks arrayed in a ring. Thus, for convenience, we henceforth refer to $X_{j n}$ (or $T_{j n}$ ) as a shift of order $n$.

In previous work [28], only one component was shifted with respect to the others, which meant the structures were relatively easy to predict. With this restriction, an order $n$ circular cluster forms only if $X_{j n}$ or $T_{j n}$ is nonzero. A higher-order shift does nothing to perturb a rogue wave, while a lower-order shift strips the ring entirely, leaving behind a rogue wave of order $n-2$. We now extend the investigation to include manipulations of several components at the same time.

General solutions of up to order 2 are analytically attainable with symbolic software, but those of higher order become computationally intractable and cumbersome to express. Instead, we numerically employ the Darboux method as a recursive algorithm. Although technically we can present exact rogue wave solutions explicitly, in reality these solutions are much too oversized to be printed in journal pages. On the other hand, the shifts can be defined as functions of $\kappa$ such that, if the structure of a certain wave function remains unchanged as $\kappa$ becomes arbitrarily small, the numerical solution can be considered to approximate a rogue wave with the shifts as free parameters of the solution. This is an efficient way of revealing the new higher-order rogue wave structures.

We emphasize here that all the results presented in the next section use the exact same method as in previous work [28]. The new physics lies instead in an updated appreciation of Eq. (2). Prior thought assumed that each component shift was equivalent to one additional degree of freedom in the rogue wave limit. Correspondingly, an order $n$ wave function only depended on $X_{j n}$ (and $T_{j n}$ ) to transform into a nontrivial circular cluster structure, while all other terms in the shift expansion were considered unimportant due to complicated cancellations in the mathematics of the Darboux procedure. It is now understood, guided by numerics, that each shift order relates to its own degree of freedom in the rogue wave limit, and the structures presented in the following section differ intrinsically from circular clusters. 


\section{THE GENERAL PRESCRIPTION FOR CASCADE SOLUTIONS}

With component shifts defined as in Eq. (2), first-order rogue waves are easy to understand. They are represented by a single Peregrine soliton that can be translated anywhere in the $(x, t)$ plane using our parameters $X_{11}$ and $T_{11}$. Second-order rogue waves also appear to be fully understood; the first-order shifts $X_{j 1}$ and $T_{j 1}$ translate the global structure, provided they are the same across the components, while the second-order shifts $X_{j 2}$ and $T_{j 2}$ break the structure apart into a triplet [21].

Complications start with the third-order rogue waves. These have three pertinent orders of shift, namely $X_{j 1}, X_{j 2}$, and $X_{j 3}$, as well as three components to move around. First-order shifts remain important only as translation variables for the global structure, and the third-order shifts appear to only separate a circular ring of five quanta from the main structure. As for the second-order shifts, they appear irrelevant when applied to one component alone [28] and, in most cases, equally unimportant when applied to multiple components. In the $\kappa \rightarrow 0$ limit, nonzero $X_{j 2}$ and $T_{j 2}$ values generally appear to expel five quanta to the infinity boundary of the $(x, t)$ plane.

However, there is a nontrivial exception to the rule for these second-order shifts. Certain combinations of values can in fact arrange the solution into a stable structure with finite circumradius, where all six quanta are visible in the domain. For simplicity, we work only with shifts along the $x$ axis and assume $X_{12}<X_{22}<X_{32}$, although the following results are easily generalized. If the three components are equally and sufficiently "spaced apart," such that $\mid X_{12}-$ $X_{22}|=| X_{32}-X_{22} \mid \gg 0$, and the frequency ratio adheres to the rule $\kappa_{1}: \kappa_{2}: \kappa_{3}=k_{1}: \sqrt{\left(k_{1}^{2}+k_{3}^{2}\right) / 2}: k_{3}$, the third-order rogue wave becomes arrayed in triangular fashion, as seen in Fig. 3(a). The six constituent quanta are well separated and the distances between the adjacent peaks are the same. We call this a rogue wave cascade as, in this typical arrangement, an observer would witness an increasing number of rogue waves over time, beginning with one solitary peak and ending with $n$ Peregrine solitons for an order $n$ structure. Of course, "cascade" is merely a convenient label. As we show later in Sec. IV, this structure can be freely rotated. This means that the progression of incidents can occur in reverse or even that the most dangerous time to experience this event may be midway between the first and last quanta observed.

With regard to structural control, varying the value of "differential shifts" tunes the spacing between the peaks. Knowing that a value of zero for all these second-order shifts results in a typical third-order rogue wave, structurally similar to Fig. 2(a) with one central high-amplitude peak, it is evident that small differential shifts will result in transitional wave functions, such as in Fig. 3(b). The triangular array is still evident in this case, but the quanta are deforming from perfect Peregrine solitons as they nonlinearly interact and merge. However, it is clear from the numerics that shifts alone do not control the interpeak spacing. As the difference between $k_{1}$ and $k_{3}$ increases, the cascade becomes more spatiotemporally compressed. Likewise, the cascade expands in the domain as the frequencies become more similar in value. The reason for this is likely to be that an $\mathrm{AB}$ with higher frequency contains more peaks within a set length than a lower-frequency

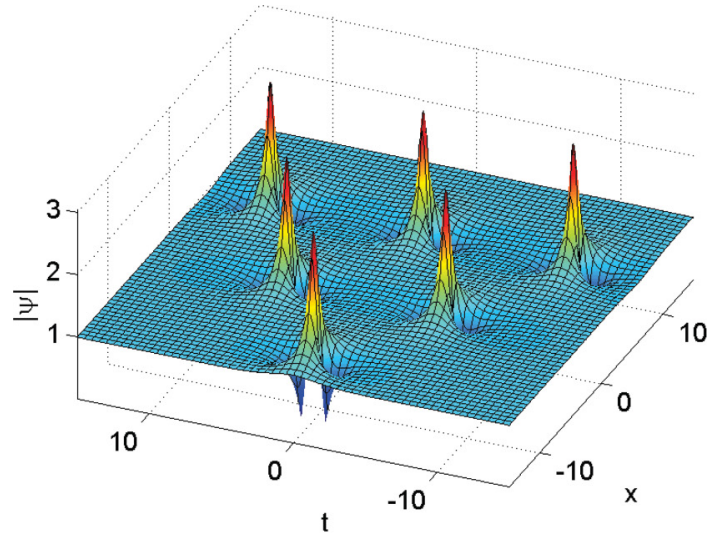

(a)

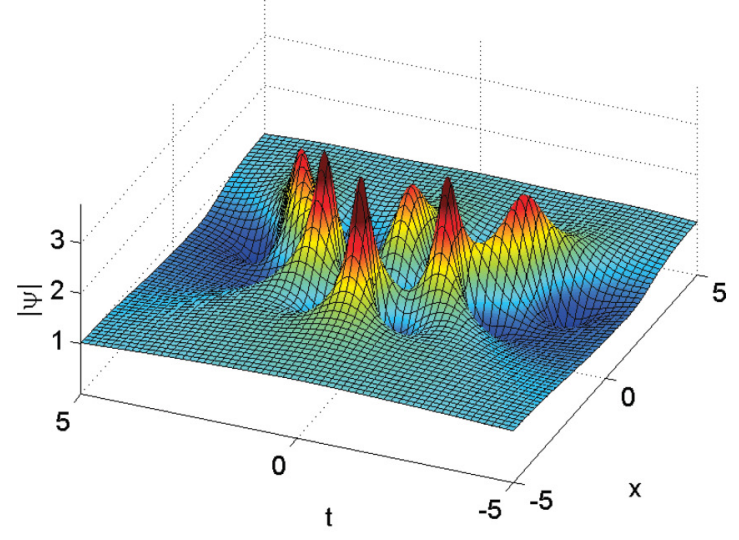

(b)

FIG. 3. (Color online) Third-order rogue wave cascades. Component frequencies $\kappa_{1}, \kappa_{2}$, and $\kappa_{3}$ have a ratio of $1: \sqrt{2}: \sqrt{3}$. (a) Three components involving six rogue wave quanta are well separated: the shifts are $X_{12}=-25, X_{22}=0$, and $X_{32}=25$. (b) The three components are overlapping: the shifts are $X_{12}=-0.25, X_{22}=0$, and $X_{32}=0.25$.

AB. Even though the rogue wave limit leaves behind only a small number of peaks, depending on component number, the proportions of the frequency ratio are still reflected in the spatiotemporal length of a cascade edge.

Remarkably, the differences $\left|X_{12}-X_{22}\right|$ and $\left|X_{32}-X_{22}\right|$ do not have to be one to one for a rogue wave cascade to arise, despite the regularity in interpeak spacing. If $d_{j}$ denotes the second-order differential shift between the components $j$ and $j+1$, then it is possible to generalize the frequency ratio required for such a structure as

$$
\kappa_{1}: \kappa_{2}: \kappa_{3}=k_{1}: \sqrt{\frac{d_{2} k_{1}^{2}+d_{1} k_{3}^{2}}{d_{1}+d_{2}}}: k_{3} .
$$

For a rogue wave cascade to exist, there must be a perfect balance between shift parameters and the frequency ratio. Thus, for example, the set of parameters $X_{12}=-300, X_{22}=$ $0, X_{32}=500$, and $\kappa_{1}: \kappa_{2}: \kappa_{3}=3: \sqrt{15}: 5$ produces a rogue wave cascade in the $\kappa \rightarrow 0$ limit, appearing no different from Fig. 3(a) except in spatiotemporal size. But any deviation from 
this prescription results in a structural transition from cascade to cluster, which is instantaneous in the $\kappa \rightarrow 0$ limit. As previously recognized [28], maintaining nonzero second-order shifts in this case forces a cluster ring to expand to the infinity horizon of the $x$ and $t$ axis, leaving behind one central Peregrine soliton. Therefore each rogue wave cascade is a specific case in parameter space and is highly unstable to general perturbation.

As mentioned by Ohta and Yang [29], higher-order cascades are also possible. For the Darboux method, this requires nothing more than a minor extension of the third-order prescription in Eq. (3). For simplicity, we continue to work only with $x$ shifts and the ordering $X_{12}<X_{22}<\cdots<X_{n 2}$, noting that the results can be generalized to include $t$ shifts and rewritten for unorthodox orderings. Recalling that $d_{j}$ is the second-order differential shift between components $j$ and $j+1$, a rogue wave cascade is always produced in the $\kappa \rightarrow 0$ limit, provided that

$\kappa_{j}: \kappa_{j+1}: \kappa_{j+2}=k_{j}: k_{j+1}: \sqrt{\frac{\left(d_{j}+d_{j+1}\right) k_{j+1}^{2}-d_{j+1} k_{j}^{2}}{d_{j}}}$,

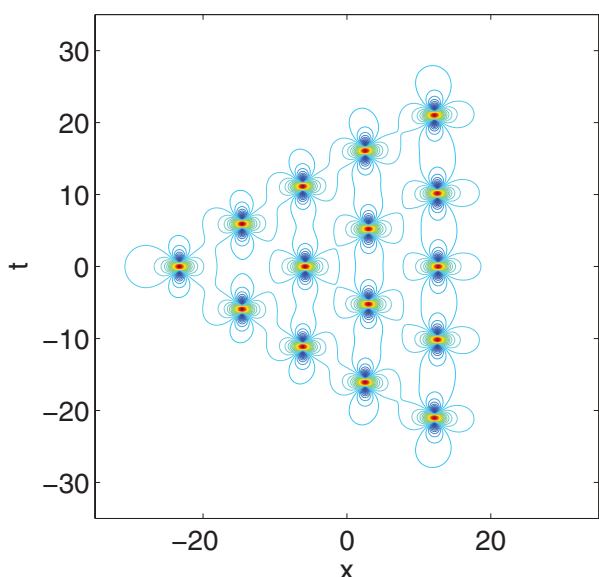

(a)

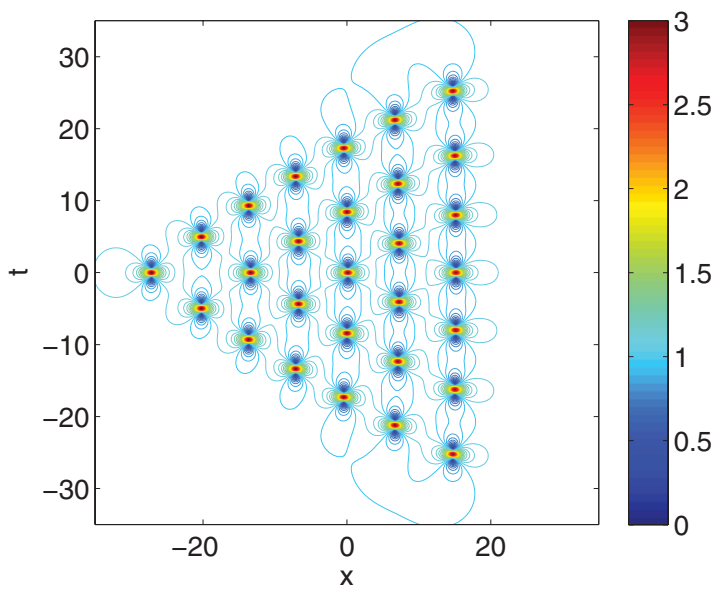

(b)

FIG. 4. (Color online) Higher-order rogue wave cascades generated recursively with Eq. (4). (a) Cascade of order 5 with $X_{j 2}=25 j^{2}$ and $\kappa_{1}: \kappa_{2}=1: \sqrt{3}$. (b) Cascade of order 7 with $X_{j 2}=25(j-4)$ and $\kappa_{1}: \kappa_{2}=1: \sqrt{2}$. for all $j$ ranging from 1 to $n-2$. This is the exact same ratio as given for the third-order case, only expressed in terms of the first two component frequencies. This recursive relation also indicates that the structural stability of a rogue wave cascade depends only on how the frequency and position of a component relates to its nearest neighbors. Provided this constraint holds, cascades are generated instead of clusters, such as the fifth-order example in Fig. 4(a) and the seventhorder example in Fig. 4(b). Curiously, the sides of the triangular arrays hint at some degree of curvature.

\section{CASCADE PERTURBATIONS AND "CLAWLIKE" STRUCTURES}

So far we have established that, modified in isolation, shifts of order $n$ generate circular clusters and second-order shifts are responsible for cascade solutions, provided a perfect balance is achieved with component frequency ratios. This is consistent with a second-order rogue wave, as a rogue triplet can be considered as both a cluster and cascade simultaneously. However, new third-order rogue wave structures, previously unreported in the literature, can be produced by manipulating shifts of second and third order simultaneously. For example, beginning with the cascade in Fig. 3(a), adding a third-order

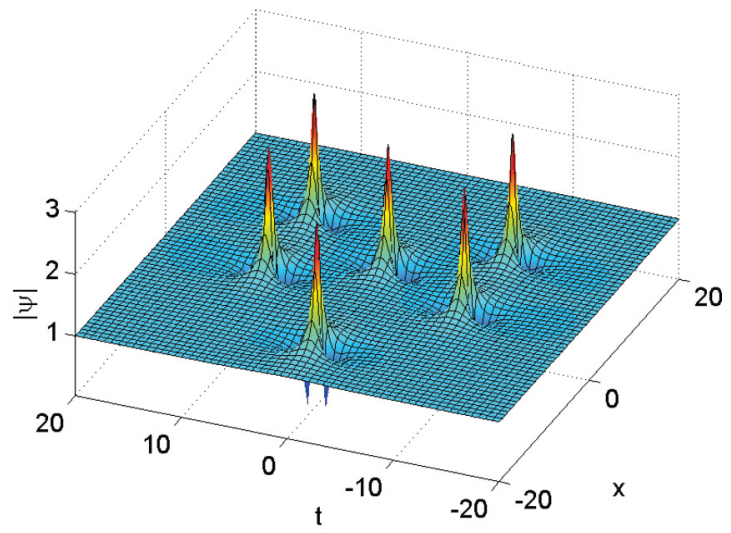

(a)

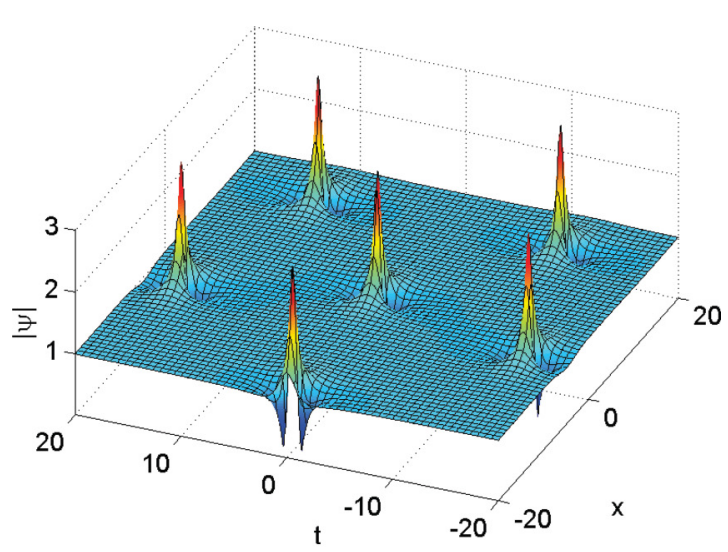

(b)

FIG. 5. (Color online) Perturbations of a third-order rogue wave cascade with $X_{12}=-25, X_{22}=0, X_{32}=25$, and $\kappa_{1}: \kappa_{2}: \kappa_{3}=1$ : $\sqrt{2}: \sqrt{3}$. (a) An "arrow" structure with $X_{23}=250$. (b) A circular cluster pointing in the negative $x$ direction with $X_{23}=2500$. 
shift to a component can deform the triangular array into an "arrow" arrangement, shown in Fig. 5(a), where three rogue wave pairs are arranged in parallel. Continuing to increase this third-order shift eventually draws one of the rogue wave quanta into the center, forming the familiar cluster shown in Fig. 5(b). In fact, larger values beyond this point will simply expand the ring of the cluster as if there were never a rogue wave cascade. This implies that structures produced by shifts of different order can coexist, but one will dominate the NLSE solution, depending on parameter values. In our previous work [28], we did not generate rogue wave cascades, so a cluster was free to grow from a central high-order peak. However, if a rogue wave cascade has been successfully produced by the right ratio of modulation frequencies and second-order shifts, then a third-order shift must be sufficiently large in order to perturb the cascade into a cluster.

The structures shown in Fig. 5 indicate how a cascade transforms into a cluster when both are "pointed" in the same direction. This requires nothing more unintuitive than a minor rearrangement of rogue wave quanta in the domain. However, if the third-order shift applied to the solution in Fig. 3(a) is

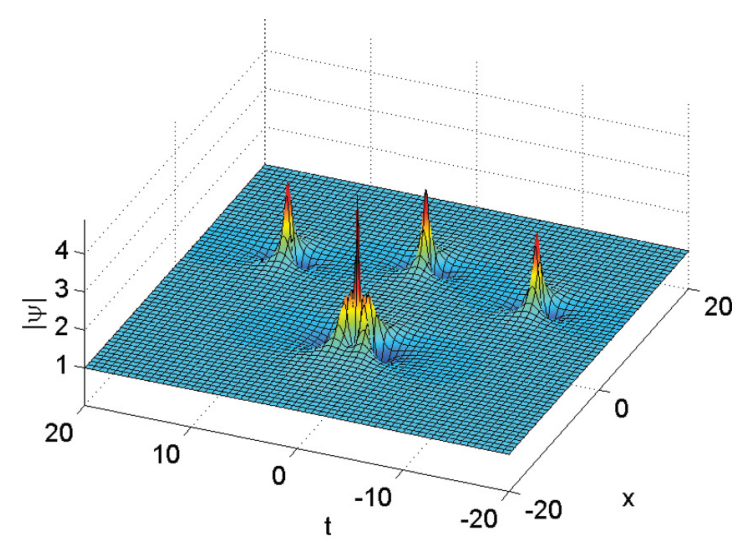

(a)

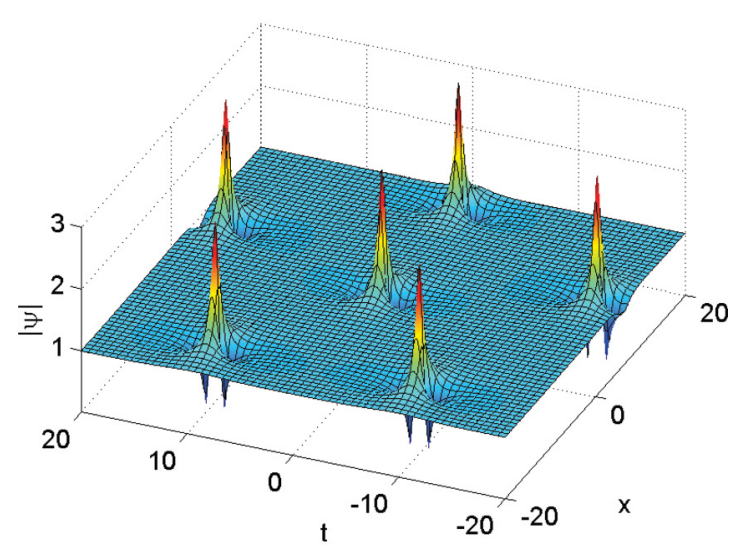

(b)

FIG. 6. (Color online) Perturbations of a third-order rogue wave cascade with $X_{12}=-25, X_{22}=0, X_{32}=25$, and $\kappa_{1}: \kappa_{2}: \kappa_{3}=1$ : $\sqrt{2}: \sqrt{3}$. (a) A "claw" structure with $X_{23} \approx-162.25$. This value correctly generates the solution numerically when $\kappa=8 \times 10^{-3}$. (b) A circular cluster pointing in the positive $x$ direction with $X_{23}=-2500$. reversed in sign, the three peaks located in the $x<0$ half-plane must eventually flip with respect to the $t$ axis in order to form a cluster that is a reflection of the one in Fig. 5(b). To achieve this, the three rogue wave quanta must merge into a second-order rogue wave, as shown in Fig. 6(a). We caution that the shift value at which this transition occurs is critically dependent on the proximity of $\kappa$ to zero in the numerical process. Thus, we provide the value of $\kappa$ used whenever detailing this transitional structure, which is unnecessary in all other cases. Nonetheless, this is a legitimate new solution of the NLSE, and we refer to it as a "claw" structure due to its spatiotemporal appearance. Furthermore, in some respects this solution is also a semicircular cluster; it has three peaks more than a solitary second-order rogue wave, all provided by the third component, but is four peaks short of a fourth-order cluster, which would be introduced by a fourth component. It is, thus, to be expected that claw structures of higher order also exist, albeit with more numerous "digits." Regardless, a sufficiently large third-order shift once again completely converts the cascade into a circular cluster, shown in Fig. 6(b), at which point any increase of shift magnitude will extend the radius of the ring.

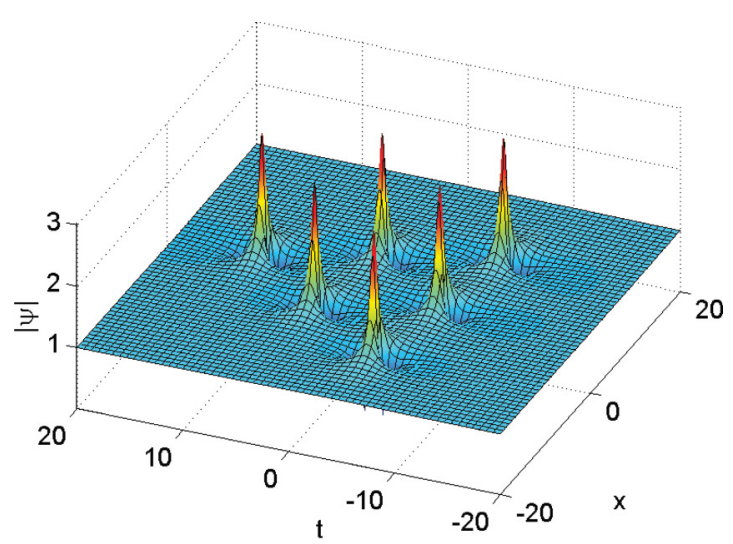

(a)

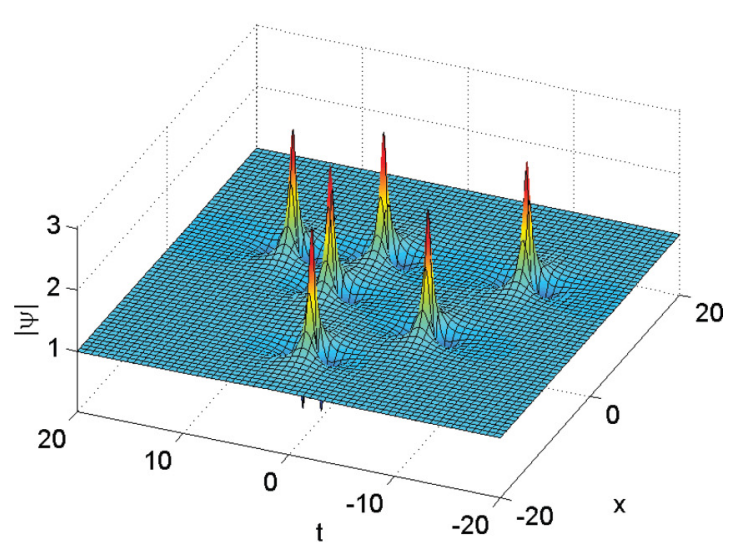

(b)

FIG. 7. (Color online) Third-order rogue wave cascades incorporating $t$-axis shifts, with $\kappa_{1}: \kappa_{2}: \kappa_{3}=1: \sqrt{2}: \sqrt{3}$. (a) A rotation with $X_{j 2}=25(j-2) \cos (\pi / 3)$ and $T_{j 2}=25(j-2) \sin (\pi / 3)$. (b) A perturbation with $X_{j 2}=25(j-2)$ and $T_{23}=-125$. 
As previously mentioned, the cascade solution is precariously dependent on the ratio of second-order shifts. It is possible to include $t$ axis shifts of this order as well, but the vectorial directions of all second-order shifts must be aligned. If this is done, the rogue wave cascade can be spatiotemporally rotated to point in any direction, as shown in Fig. 7(a). However, no such restriction exists for third-order shifts. Hence, a cascade formed by second-order $x$-axis shifts can be perturbed by third-order $t$-axis shifts. This opens up a new range of asymmetrical rogue wave solutions, such as is shown in Fig. 7(b). As usual, a sufficiently large third-order shift will expand the structure into a circular cluster, neutralizing the effect of any second-order shifts.

By this stage, the possible variety of third-order rogue wave solutions is reasonably well understood. However, each additional increase of solution order introduces a greater degree of complexity. From the examples in Fig. 4, it is evident that second-order shifts can produce cascade solutions of any order. It also follows that a shift of order $n$ applied

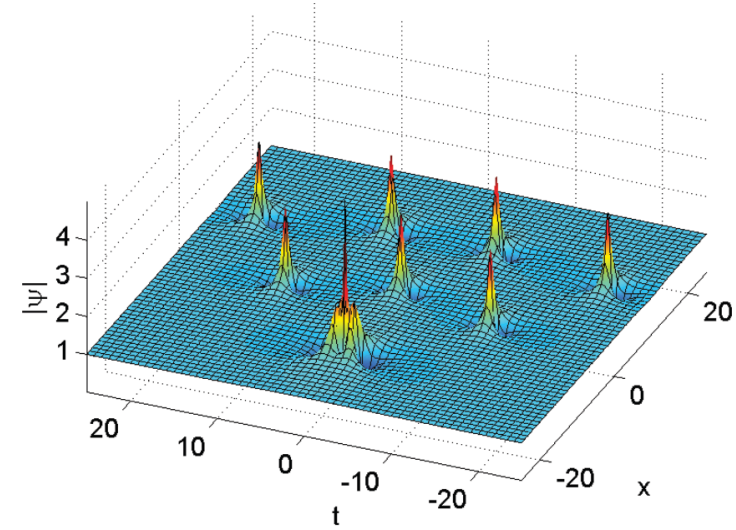

(a)

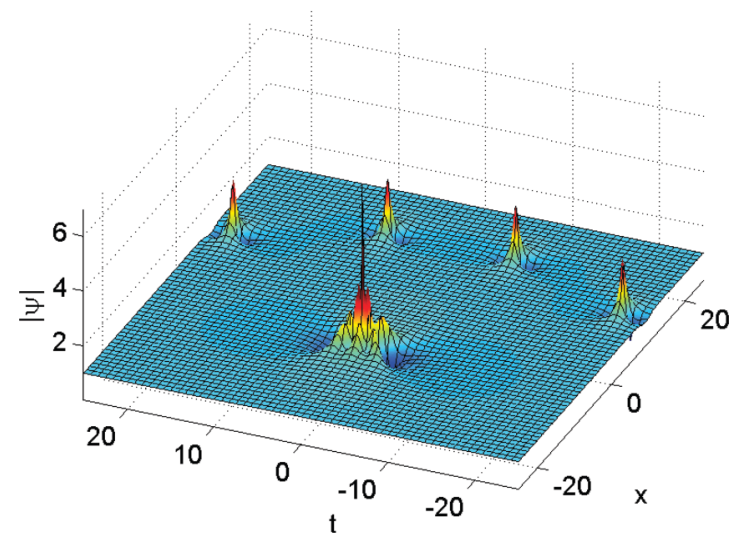

(b)

FIG. 8. (Color online) Fourth-order rogue wave claws with $X_{12}=-75, X_{22}=-25, X_{32}=25, X_{42}=75$, and $\kappa_{1}: \kappa_{2}: \kappa_{3}: \kappa_{4}=$ $1: \sqrt{2}: \sqrt{3}: \sqrt{4}$. (a) Two rows of rogue wave quanta, where $X_{23}=X_{33} \approx-425$. These values correctly generate the solution numerically when $\kappa=8 \times 10^{-3}$. (b) One row of rogue wave quanta, where $X_{23}=X_{33} \approx-1042.5$ and $X_{34} \approx-13100$. These values correctly generate the solution numerically when $\kappa=2 \times 10^{-2}$. to any component can perturb the structure and still produce a $\kappa \rightarrow 0$ rogue wave solution of the NLSE. However, the intermediate orders of shift are also expected to produce novel and elegantly arranged structures. Even without cataloguing all these solutions, we can still show numerically that the results of the Darboux process adhere to certain patterns.

Recognizing that the clawlike structure in Fig. 6(a) was generated by applying a third-order shift to the middle component so it approached the first, the middle two components of a fourth-order cascade can similarly be pushed towards the apex. In this process, three quanta belonging to two components merge and form a second-order rogue wave. The entire solution, shown in Fig. 8(a), looks identical to the claw in Fig. 6(a), except for an additional four rogue wave quanta that form a second row. Furthermore, by applying a fourth-order shift to the structure, specifically pushing the third component towards the first two, it is possible to construct a claw with only one row of quanta, shown in Fig. 8(b). The trade-off is that the focal peak transforms from a second-order to a third-order rogue wave, due to fusion with three extra rogue wave quanta. Thus, we show that rogue claw structures are generic across all orders of nonlinear superposition and display predictable features.

\section{CONCLUSION}

In summary, our main results are as follows:

(1) We have shown the existence of a third-order triangularly arrayed multi-rogue-wave solution, generated numerically with the recursive Darboux method. These have recently been found as particular examples by Ohta and Yang [29], although we have further identified a general prescription for these "cascade" solutions, which involves prelimit component frequencies and second-order shifts.

(2) We have extended the prescription such that the Darboux method allows us to produce rogue wave cascades of any order. In all such cases, the shifts required are still of second order. Furthermore, as shifts of order $n$ produce circular clusters for an order $n$ solution, this suggests that shift orders between 2 and $n$ may be responsible for their own as-yet-undiscovered unique structures.

(3) We have further explored third-order rogue wave cascades and presented new solutions of the NLSE by including third-order terms in shifts. While the cascade prescription rigidly restricts the choice of second-order terms in shifts, the third-order terms are independent and can generate a myriad of perturbed forms. The relative strength of second-order shifts with respect to third-order shifts determines how similar the hybrid structure is to a cascade or cluster.

(4) We have shown that the new forms are not trivial and that Peregrine soliton peaks can merge to produce rather unexpected arrangements. In particular, we reveal new clawlike structures. These can also be considered as "semicircular" clusters and are indicative of an alternative class in the cluster hierarchy.

From a geometrical perspective, rogue wave cascades enrich the pattern of higher-order NLSE solutions. It is remarkable that the particular choice of NLSE in Eq. (1) produces limiting breather solutions that include not only circularly symmetric arrangements, but arrays in the shape 
of equilateral triangles as well. Furthermore, these structures are all associated with certain orders of shift in Eq. (2). This allows us to speculate that other elegant arrangements may also be possible for rogue waves of order 4 and beyond, simply by constraining relative component frequencies and other orders of shift in some manner. These prescriptions can only be proved beyond doubt by analytics, but the fact that these relations and their effect on structure are numerically well evident implies that a good understanding has been achieved already.

With regard to experiment, cascade solutions are important as the first theorized rogue wave structures beyond order 3 that do not contain any substructures other than Peregrine solitons. This means that experimentalists can produce highorder arrangements without facing unduly large amplitudes. This is particularly beneficial in water wave tanks $[18,19]$ as it is thought that wave breaking may limit the crest height with respect to the pulse width [37]. The scaling transform [20] can alleviate some of the pressure from breaking by decreasing the solution amplitude, but this comes at the cost of increasing the spatiotemporal width, which is not ideal in a tank of finite length. For this reason, it is expected that it will be easier to produce cascades than circular clusters in water.

The triangular arrangement of first-order rogue waves in a cascade may also be important in optics. In one sense, such a structure has already been seen in fibre, arising from a perturbed plane wave due to beating laser frequencies [38]. Despite minor variations in shape and the unavoidable periodicity of experimental pulse sequences, the triangular nature of the observed arrays is unmistakable. These observations are naturally linked to higher-order modulation instability. However, it will not be surprising if an ideal cascade emerges from increasing the beat period, much as an Akhmediev breather served as a pragmatic forerunner of an ideal rogue wave [17]. Furthermore, this triangular symmetry may be far more fundamental to nonlinear optics in general, with similar shapes produced experimentally from wave interactions in transverse pattern systems [39,40].

In any case, the increased number of peaks in a rogue wave cascade relative to that of circular cluster could prove relevant to the real world in its own way. If encountered in the ocean, an order $n$ rogue wave cascade has $n(n+1) / 2$ chances to impact a vessel, as opposed to $2 n$ chances for a circular cluster (where $n>2$ ). Worse yet, if the event is first encountered at a spatiotemporal vertex, the sea will only become rougher. Furthermore, considering that the possibility of peaks arising stochastically becomes smaller for higher orders, a cascade may be the typical form of complex rogue wave encountered in the ocean, much like the ubiquitous "three sisters" phenomenon. As for photonics, producing a cascade may be a way to transmit signals produced by the same initial condition to different locations along the fiber. Alternatively, rogue wave cascades could be used to load-test materials in progressive steps with intense pulses. Whatever the eventual application, a detailed knowledge of possible rogue wave superpositions is no less important than that of multi-soliton solutions. While we have a complete understanding of the latter, conveniently aided by solitonic persistence in the time domain, multi-rogue-wave solutions are far more diverse and still require significant efforts to both comprehend and categorize.

In conclusion, we have studied a subset of higher-order NLSE rogue wave solutions that manifests itself as a triangular array of first-order rogue waves. We have also shown that the free real parameters that govern these structures are independent from those that produce circular rogue wave clusters. With particular emphasis on third-order structures, we have shown that careful modification of all parameters can produce cluster, claw-like and cascade structures, with a wide variety of intermediate patterns included. In this way, we move yet another step closer to understanding the full hierarchy of NLSE rogue wave solutions, which progressively appears to be much more complicated than the painfully familiar world of NLSE solitons.

\section{ACKNOWLEDGMENTS}

The authors acknowledge the support of the Australian Research Council (Discovery Project number DP110102068). N.A. and A.A. acknowledge support from the Volkswagen Stiftung. N.A. acknowledges financial support from an Alexander von Humboldt Award (Germany).
[1] P. Müller, C. Garrett, and A. Osborne, Oceanography 18, 66 (2005).

[2] I. Nikolkina and I. Didenkulova, Nat. Hazards: Earth Syst. Sci. 11, 2913 (2011).

[3] E. Pelinovsky, E. Shurgalina, and N. Chaikovskaya, Nat. Hazards Earth Syst. Sci. 11, 127 (2011).

[4] I. Didenkulova, Boreal Environ. Res. 16, 138 (2011).

[5] K. B. Dysthe and K. Trulsen, Phys. Scr., T 82, 48 (1999).

[6] N. Akhmediev, A. Ankiewicz, and J. M. Soto-Crespo, Phys. Rev. E 80, 026601 (2009).

[7] H. S. Chiu, K. W. Chow, and C. H. Tsang, in Proceedings of the 33rd IAHR Congress, 2009 (Curran Associates, Inc., Red Hook, NY, 2010), pp. 6232-6239.

[8] A. R. Osborne, Nonlinear Ocean Waves and the Inverse Scattering Transform (Elsevier, Amsterdam, 2010).
[9] E. Pelinovsky and C. Kharif, Extreme Ocean Waves (Springer, Berlin, 2008).

[10] C. Garrett and J. Gemmrich, Phys. Today 62, 62 (2009).

[11] D. R. Solli, C. Ropers, P. Koonath, and B. Jalali, Nature 450, 1054 (2007).

[12] M. Shats, H. Punzmann, and H. Xia, Phys. Rev. Lett. 104, 104503 (2010).

[13] V. B. Efimov, A. N. Ganshin, G. Kolmakov, P. McClintock, and L. Mezhov-Deglin, Eur. Phys. J. Special Topics 185, 181193 (2010).

[14] Y. V. Bludov, V. V. Konotop, and N. Akhmediev, Phys. Rev. A 80, 033610 (2009).

[15] D. Peregrine, J. Austral. Math. Soc. Ser. B 25, 16 (1983).

[16] V. Shrira and V. Geogjaev, J. Eng. Math. 67, 11 (2010).

[17] B. Kibler, J. Fatome, C. Finot, G. Millot, F. Dias, G. Genty, N. Akhmediev, and J. M. Dudley, Nat. Phys. 6, 790 (2010). 
[18] A. Chabchoub, N. P. Hoffmann, and N. Akhmediev, Phys. Rev. Lett. 106, 204502 (2011).

[19] A. Chabchoub, N. Hoffmann, M. Onorato, and N. Akhmediev, Phys. Rev. X 2, 011015 (2012).

[20] N. Akhmediev, A. Ankiewicz, and M. Taki, Phys. Lett. A 373, 675 (2009).

[21] A. Ankiewicz, D. J. Kedziora, and N. Akhmediev, Phys. Lett. A 375, 2782 (2011).

[22] A. Ankiewicz, P. Clarkson, and N. Akhmediev, J. Phys. A-Math. Theor. 43, 122002 (2010).

[23] C. Kharif and E. Pelinovsky, Eur. J. Mech. B-Fluids 22, 603 (2003).

[24] K. Dysthe, H. E. Krogstad, and P. Müller, Annu. Rev. Fluid Mech. 40, 287 (2008).

[25] F. T. Arecchi, U. Bortolozzo, A. Montina, and S. Residori, Phys. Rev. Lett. 106, 153901 (2011).

[26] P. Dubard, P. Gaillard, C. Klein, and V. Matveev, Eur. Phys. J. Spec. Top. 185, 247 (2010).

[27] P. Gaillard, J. Phys. A 44, 5204 (2011).

[28] D. J. Kedziora, A. Ankiewicz, and N. Akhmediev, Phys. Rev. E 84, 056611 (2011).

[29] Y. Ohta and J. Yang, Royal Soc. London Proc. Ser. A 468, 1716 (2012).
[30] P. Dubard and V. B. Matveev, Nat. Hazards Earth Sys. 11, 667 (2011).

[31] V. B. Matveev and M. Salle, Darboux Transformations and Solitons (Springer-Verlag, Berlin, 1991).

[32] N. Akhmediev and N. V. Mitskevich, IEEE J. Quantum Electron. 27, 849 (1991).

[33] N. Akhmediev, J. M. Soto-Crespo, and A. Ankiewicz, Phys. Lett. A 373, 2137 (2009).

[34] H. C. Yuen and B. M. Lake, Ann. Rev. Fluid Mech. 12, 303 (1980).

[35] D. J. Kedziora, A. Ankiewicz, and N. Akhmediev, Phys. Rev. E 85, 066601 (2012).

[36] P. Gaillard (2012), preprint: hal-00664052, version 1-28 Jan 2012.

[37] A. V. Babanin, T. Waseda, I. Shugan, and H.-H. Hwung, ASME Conf. Proc. 2, 409 (2011).

[38] M. Erkintalo, K. Hammani, B. Kibler, C. Finot, N. Akhmediev, J. M. Dudley, and G. Genty, Phys. Rev. Lett. 107, 253901 (2011).

[39] U. Bortolozzo, L. Pastur, P. L. Ramazza, M. Tlidi, and G. Kozyreff, Phys. Rev. Lett. 93, 253901 (2004).

[40] P. L. Ramazza, U. Bortolozzo, and L. Pastur, J. Opt. A: Pure Appl. Opt. 6, S266 (2004). 